\title{
CORAL REEF ANTHROPOGENIC IMPACT BIO-INDICATORS IN THE NORTHERN PART OF THE PERSIAN GULF
}

\author{
H. VALAVI ${ }^{1}$ - A. SAVARI ${ }^{1}-$ V YAVARI $^{2}-$ P. KOCHANIAN $^{2}-$ A. SAFAHIEH ${ }^{1}-$ O.SEDIGHI \\ SAVADKUHI \\ ${ }^{1}$ Department of Marine Biology, Faculty of Marine Science, Khorramshahr University of \\ Marine Science and Technology, P.O. Box: 669, Khorramshahr, Khuzestan, Iran. (Phone: +98- \\ 9177169337; fax: +98-7112318630) \\ ${ }^{2}$ Department of Fisheries, Faculty of Natural Resources, Khorramshahr University of Marine \\ Science and Technology, P.O. Box: 669, Khorramshahr, Khuzestan, Iran. (Phone: +98- \\ 6324233322; fax: +98-6324233322) \\ ${ }^{3}$ Department of the Environment, P.O. Box: 5181, Tehran, Iran. (Phone: +98-2188244570; fax: \\ $+98-2188244570$ ) \\ *Corresponding author \\ e-mail: hamzehvalavi@yahoo.com \\ (Received $13^{\text {th }}$ October 2009 ; accepted $13^{\text {th }}$ November 2009)
}

\begin{abstract}
Potential and efficiency of coral reef bio-indicators proposed by Reef Check for coral reef monitoring in the Persian Gulf were studied as anthropogenic impact bio-indicators. Data were collected from the coral reefs in the northern part of the Persian Gulf in 2007 using Reef Cheek standard methodology and analyzed using Redundancy Analysis and Indicator Species Analysis. Similar data collected in 2002 and 2003 were also incorporated into our data. According to the results short-spine sea urchin (Echinometra mathaei) showed consistent positive correlation with commercial fishing and high indicator value for commercial fishing areas and could be pointed out as a weak bio-indicator of overfishing. Also Arabian butterfly fish (Chaetodon melapterus), showed negative correlation with commercial fishing and high significant indicator values for none to low fishing areas in 2003 and 2007 and could be considered as indicator of low fishing pressure in the region. None of Fin fishes proposed as indicators of over-fishing, and also other proposed species showed consistent correlation or consistent significant indicator values for any anthropogenic impacts and are not recognized as anthropogenic impact bio-indicators. It is concluded that a much shorter and more efficient list of bio-indicators could be used for monitoring coral reefs in this region.
\end{abstract}

Key words: Reef Check, IndicatorSpecies, Overfishing, Arabian butterfly fish, Short-spine sea urchin.

\section{Introduction}

Coral reef communities in the Persian Gulf exist in a harsh environment with respect to salinities, sea temperatures and extreme low tides [6]. In this region, coral reefs experience long periods (months) of high sea temperature (above $30{ }^{\circ} \mathrm{C}$ ) in the summer and low (below $16{ }^{\circ} \mathrm{C}$ ) temperature in the winter [9].

These factors have a serious influence on community structure by restricting the number of species in the area and by causing recurrent mortality among the dominant species $[6,11$,$] . In last two decades, coral bleaching has occurred throughout the world$ resulting in mass mortality of corals mainly due to the elevated temperature [28]. This has also been the case in the Persian Gulf [24].

However, to date, the majority of damage to coral reefs around the world including Persian Gulf and Iranian waters has been through direct anthropogenic stress [2, 4, 8, $12,14,15,23,26]$. The major causes of damage are: 
Excessive pollution from domestic, industrial and agricultural waste; poor land use practices, which increase the amount of land derived sediments flowing onto coral reefs; and over exploitation, particularly through damaging practices such as dynamite fishing.

Industrial projects could cause such anthropogenic damage to coral reefs mainly through industrial waste pollution, poor land use practice, uncontrolled coastal construction and reclamation.

Reef Check (RC), as the largest and most widespread global organization dedicated to monitoring reefs proposes some fish and invertebrate indicators for coral reef monitoring programe in the Persian Gulf region [13].

These indicators include: barramundi cod (Cromileptes altivelis), orange-spotted grouper (Epinephelus coioides), other groupers, grey grunt (Plectorhinchus sordidus), black-spotted grunt (Plectorhinchus gaterinus), spotted grunt (Plectorhinchus pictus), dark butterfly fish (Chaetodon nigropunctatus), Arabian butterfly fish (Chaetodon melapterus), long-fin butterfly fish (Heniochus acuminatus), grunts/Sweetlips, parrot fish, snappers, moray eel (all species), humphead wrasse (Cheilinus undulates), longspined black sea urchin (Diadema spp.) banded coral shrimp (Stenopus hispidus), lobster (all edible species), collector sea urchin (Tripneustes spp.), black sea urchin (Echinothrix diadema), cowries, pencil sea urchin (Heterocentrotus mammilatus), shortspine sea urchin (Echinometra mathaei), crown-of-thorns starfish (Acanthaster planci), edible sea cucumbers (e.g. teatfish Holothuria nobilis), Triton (Charonia tritonis).

It is very necessary to mention here that there are some serious problems in using bio-indicator-dependent monitoring programs. Firstly when management goals are indistinct and unclear wrong variables may be monitored and therefore inappropriate indicators may be selected, secondly relying on inappropriate bio-indicators fails to reflect changes in environment and leads to poor management, and finally in many cases enough scientific effort is not applied to select bio-indicators and in other words there is a 'lack of robust procedures for selecting ecological indicators' [3].

Finally each monitoring problem requires individual treatment and 'there is no one bio-indicator species that will suit all programmes' [16]. The complexity and variety of reef ecosystems makes it very difficult to confidently select a single indicator for a large region. Therefore the purpose of this study is to review coral reef fishes and invertebrates indicators proposed for the Persian Gulf region by Reef Check and determine potential and effectiveness of these indicators as anthropogenic impacts in the northern part of the Persian Gulf using Reef Check data or data collected using standard RC methodology [13].

\section{Materials and Methods}

\section{Study Area}

Data for this study were collected from the coral reefs at Khark, Kharku, Hendorabi, Kish, Farur and Farurgan Islands and Nayband bay in 2007 in the northern part of the Persian Gulf. Similar data collected in 2002 and 2003 in some of the mentioned regions and in Lavan and Larak Islands were also used (Fig. 1). 


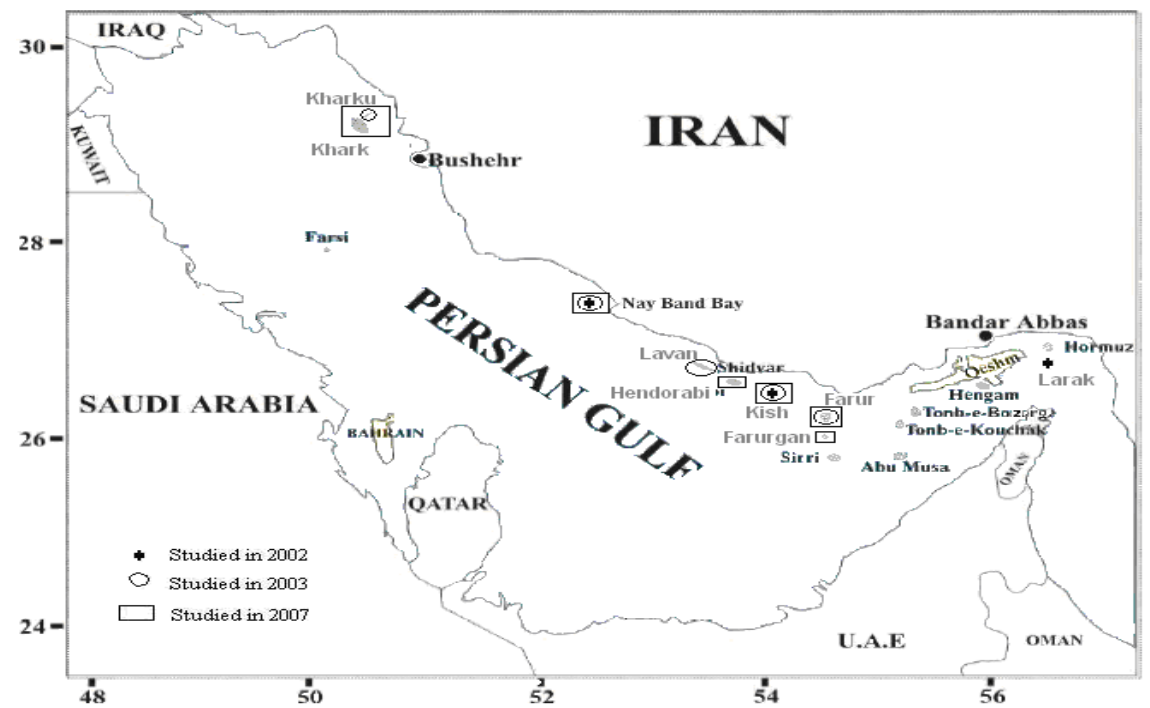

Figure 1. Study areas in 2002, 2003 \& 2007 in the northern Persian Gulf

\section{Sampling techniques}

Survey sites were chosen using manta tow surveys and reconnaissance dives. They were popular diving areas, the 'best' reefs in the area or the 'worst' reefs in the area and they reflect a wide range of habitats.

At each site a site description sheet was completed with anecdotal, observational, historical, locational and other data. This included impacts at the site, giving values of 0 (none), 1 (low), 2 (medium) or 3 (high) for the following: 'tourist diving', 'sewage pollution', 'industrial pollution', 'commercial fishing' and 'other impacts' (Table 1).

Table 1. Site description definitions and field guide, clarifying measurements.

\begin{tabular}{|c|c|c|c|}
\hline Impact & Low (1) & Medium (2) & High (3) \\
\hline Aquarium fishing & $\begin{array}{l}\text { Less than once per } \\
\text { month }\end{array}$ & $\begin{array}{l}\text { More than once per month, but } \\
\text { less than once per week }\end{array}$ & Once a week or more \\
\hline Tourist diving & $\begin{array}{l}\text { 1-5 individuals per } \\
\text { day }\end{array}$ & 6-20 individuals per day & $\begin{array}{c}\text { More than } 20 \text { individuals } \\
\text { per day }\end{array}$ \\
\hline Sewage pollution & $\begin{array}{l}\text { Sewage, irregular } \\
\text { or rare discharge }\end{array}$ & $\begin{array}{c}\text { Source of discharge }>100 \mathrm{~m} \\
\text { but }<500 \mathrm{~m} \text { from transect }\end{array}$ & $\begin{array}{c}\text { Source of discharge }<100 \\
\mathrm{~m} \text { from any point on } \\
\text { transect }\end{array}$ \\
\hline Industrial pollution & Source $>0.5 \mathrm{~km}$ & Source between 0.1 and $0.5 \mathrm{~km}$ & Source less than $100 \mathrm{~m}$ \\
\hline Commercial fishing & $\begin{array}{l}\text { Less than once per } \\
\text { month }\end{array}$ & $\begin{array}{l}\text { Less than once a week and } \\
\text { more than once a month }\end{array}$ & Once a week or less \\
\hline
\end{tabular}

Then data were collected along 2 depth contours at shallow (3-6 m) and intermediate (6-12 m) depths (if the reef was too shallow, the 6-12 $\mathrm{m}$ depth transect was not completed). Along each depth contour a 100 meter transect was placed and along it four 20 meters replicate transects were surveyed. The start and end points of 20 meters transects were five meters apart. 
Along each transect at each depth a belt transect $(5 \mathrm{~m}$ wide centered on each 20 meters transect line) was sampled for commercially important fish favored by fishers and aquarium and invertebrate taxa typically targeted for curios and food [13].

\section{Statitical Methods}

Detrended correspondence analyses (DCA) were run on the fish and invertebrate data sets using Canoco 4.0 to determine the unimodality of the data. Detrending was done by segments, species were square-root transformed and rare species were down-weighted. Following Chi-squared measure distance and one standard deviation cutoff, Outliers were identified and removed from the data set using PC-ORD 4.17 [21].

Redundancy analyses (RDA) were run using Canoco 4.0 to determine correlations between fish and invertebrate vs. anthropogenic impact variables because all DCA axis 1 gradients were below 2.5 science RDA is useful where gradients are shorter [22]. Once RDA's were performed, collinear anthropogenic variables, those with variance inflation factors (VIF) over 10, were deleted [5], also all data were checked for normality using the Anderson-Darling test in Minitab 13.20, in cases where $p$ values were below 0.05 (Non-normal distribution), data were $\log$ transformed using $\mathrm{x}=$ $\log (\mathrm{x}+1)$.

Indicator species were identified for each habitat type using the method introduced by [10] based on an indicator value index (IndVal) as follows:

$$
\text { Specificity measure : } A_{i j}=\text { Nindividuals }_{i j} / \text { Nindividuals }_{i}
$$

In our case Nindividuals $s_{i j}$ is the mean number of species $i$ across transects of group $j$, and Nindividuals $s_{i}$ is the sum of the mean numbers of individuals of species $i$ over all groups;

$$
\text { Fidelity measure }: \mathrm{B}_{j i}=\text { Nsites }_{j i} / \text { Nsites }_{j}
$$

In our case $N_{\text {sites }}$ ij is the number of transects in cluster (habitat) $j$ where species $i$ is present, and Nsites $_{j}$ is the total number of transects in that cluster.

The percentage indicator value (IV) for species $i$ in cluster (habitat) $j$ is then:

$$
\operatorname{IndVal}_{i j}=A_{i j} \times B_{j i} \times 100
$$

For maximum $A_{i j}$, species $i$ is only present in cluster $j . B_{j i}$ is highest when species $i$ is present in all transects of cluster $j$. indicator value is thus highest (100\%) when species $i$ is present in all transects of only one habitat group. The significance of the indicator values were tested using a random reallocation of transects among transects groups using Monte Carlo randomization test (1000 permutations).

The site hierarchy component of [10] method to select site clusters was not preformed because the transects were already clustered into groups based on different levels of anthropogenic impacts with the following groups: 0 (None), 1(Low), 2 (Medium) and 3 (High).

The calculations of IVs and the associated Monte Carlo (randomization) test were performed using the PC-ORD 4.17 software [21]. 


\section{Results}

\section{Abundance of indicators and levels of anthropogenic impacts}

Levels of anthropogenic impacts in transects and average abundance of indicator fish and invertebrates within belt transects studied in 2002, are respectively presented in Tables 2 and 3.

Table 2. Levels of anthropogenic impacts in 2002 transects

\begin{tabular}{c|c|c|c|c|c|c}
\hline \multirow{2}{*}{$\begin{array}{c}\text { Anthropogenic } \\
\text { impact }\end{array}$} & \multicolumn{2}{|c|}{ Kish 1 } & \multicolumn{2}{c|}{ Kish 2 } & \multicolumn{2}{c}{ Larak } \\
\cline { 2 - 6 } & $\mathbf{3 - 6 ~ m}$ & $\mathbf{6 - 1 2} \mathbf{~ m}$ & $\mathbf{3 - 6 ~} \mathbf{~ m}$ & $\mathbf{6 - 1 2} \mathbf{~ m}$ & $\mathbf{3 - 6 ~ m}$ & $\mathbf{6 - 1 2} \mathbf{~ m}$ \\
\hline Aquarium fishing & 1 & 1 & 0 & 0 & 0 & 0 \\
Commercial fishing & 1 & 1 & 0 & 0 & 1 & 0 \\
Sewage & 0 & 0 & 0 & 0 & 0 & 0 \\
Industrial pollution & 0 & 0 & 0 & 0 & 0 & 0 \\
Tourist diving & 1 & 0 & 0 & 0 & 0 & 0 \\
\hline
\end{tabular}

Table 3. Average indicator fish/invertebrate density (individual/100 $\mathrm{m}^{2}$ ) in 2002 transects

\begin{tabular}{c|c|c|c|c|c|c}
\hline \multirow{2}{*}{ Fish/Invertebrate } & \multicolumn{2}{|c|}{ Kish 1 } & \multicolumn{2}{c|}{ Kish 2 } & \multicolumn{2}{c}{ Larak } \\
\cline { 2 - 6 } & $\mathbf{3 - 6 ~ m}$ & $\mathbf{6 - 1 2} \mathbf{~ m}$ & $\mathbf{3 - 6 ~ m}$ & $\mathbf{6 - 1 2} \mathbf{~ m}$ & $\mathbf{3 - 6 ~ m}$ & $\mathbf{6 - 1 2} \mathbf{~ m}$ \\
\hline Orange-spotted grouper & 0.00 & 0.25 & 0.00 & 0.00 & 0.00 & 0.00 \\
Other groupers & 0.00 & 0.00 & 0.25 & 0.00 & 0.50 & 0.25 \\
Hump-head wrasse & 1.00 & 0.00 & 0.00 & 0.00 & 0.00 & 0.00 \\
Dark butterfly & 0.50 & 1.00 & 0.00 & 0.50 & 14.50 & 0.00 \\
Arabian butterfly & 0.25 & 0.00 & 0.00 & 0.00 & 0.50 & 0.00 \\
Long-fin butterfly & 0.00 & 1.00 & 0.75 & 0.00 & 0.00 & 0.00 \\
Parrot & 0.00 & 0.25 & 1.75 & 0.25 & 2.00 & 0.00 \\
Moray eel & 0.00 & 0.00 & 0.00 & 0.25 & 0.00 & 0.00 \\
\hline Short-spine sea urchin & 1.50 & 16.00 & 5.25 & 41.75 & 3.25 & 0.00 \\
Pencil sea urchin & 0.00 & 3.00 & 0.00 & 0.00 & 0.00 & 0.00 \\
Sea cucumber & 0.00 & 3.00 & 0.50 & 0.00 & 0.50 & 0.00 \\
Lobster & 0.00 & 0.00 & 0.25 & 0.00 & 0.00 & 0.00 \\
\hline
\end{tabular}

Similar data for 2003 are presented in Tables 4 and 5 and for 2007 in Tables 6 and 7 respectively.

Table 4. Levels of anthropogenic impacts in 2003 transects

\begin{tabular}{c|c|c|c|c|c|c|c|c|c|c|c|c|c}
\hline \multirow{2}{*}{ Anthropogenic impact } & \multicolumn{2}{|c|}{ Nayband 1 } & \multicolumn{2}{c|}{ Kharku } & \multicolumn{2}{c|}{ Lavan } & \multicolumn{2}{c|}{ Kish 1 } & Kish 2 & \multicolumn{2}{c|}{ Farur } & Nayband 2 \\
\cline { 2 - 12 } & $\begin{array}{c}\mathbf{3 - 6} \\
\mathbf{m}\end{array}$ & $\begin{array}{c}\mathbf{6 - 1 2} \\
\mathbf{m}\end{array}$ & $\begin{array}{c}\mathbf{3 - 6} \\
\mathbf{m}\end{array}$ & $\begin{array}{c}\mathbf{6 - 1 2} \\
\mathbf{m}\end{array}$ & $\begin{array}{c}\mathbf{3 - 6} \\
\mathbf{m}\end{array}$ & $\begin{array}{c}\mathbf{6 - 1 2} \\
\mathbf{m}\end{array}$ & $\begin{array}{c}\mathbf{3 - 6} \\
\mathbf{m}\end{array}$ & $\begin{array}{c}\mathbf{6 - 1 2} \\
\mathbf{m}\end{array}$ & $\begin{array}{c}\mathbf{3 - 6} \\
\mathbf{m}\end{array}$ & $\begin{array}{c}\mathbf{3 - 6} \\
\mathbf{m}\end{array}$ & $\begin{array}{c}\mathbf{6 - 1 2} \\
\mathbf{m}\end{array}$ & $\begin{array}{c}\mathbf{3 - 6} \\
\mathbf{m}\end{array}$ & $\begin{array}{c}\mathbf{6 - 1 2} \\
\mathbf{m}\end{array}$ \\
\hline Aquarium fishing & 0 & 0 & 0 & 0 & 0 & 0 & 1 & 1 & 0 & 1 & 1 & 0 & 0 \\
Commercial fishing & 3 & 3 & 2 & 2 & 2 & 2 & 1 & 1 & 2 & 1 & 1 & 3 & 3 \\
Sewage & 0 & 0 & 0 & 0 & 0 & 0 & 0 & 0 & 2 & 0 & 0 & 0 & 0 \\
Industrial pollution & 0 & 0 & 0 & 0 & 0 & 0 & 0 & 0 & 0 & 0 & 0 & 0 & 0 \\
Tourist diving & 0 & 0 & 0 & 0 & 0 & 0 & 2 & 0 & 0 & 0 & 0 & 0 & 0 \\
\hline
\end{tabular}


Table 5. Average indicator fish/invertebrate density (individual/100 $\mathrm{m}^{2}$ ) in 2003 transects

\begin{tabular}{|c|c|c|c|c|c|c|c|c|c|c|c|c|c|}
\hline \multirow[b]{2}{*}{ Fish/Invertebrate } & \multicolumn{2}{|c|}{ Nayband 1} & \multicolumn{2}{|c|}{ Kharku } & \multicolumn{2}{|c|}{ Lavan } & \multicolumn{2}{|c|}{ Kish 1} & \multirow{2}{*}{$\begin{array}{c}\text { Kish } 2 \\
3-6 \\
\text { m } \\
\end{array}$} & \multicolumn{2}{|c|}{ Farur } & \multicolumn{2}{|c|}{ Nayband 2} \\
\hline & $\begin{array}{c}\text { 3-6 } \\
\text { m }\end{array}$ & $\begin{array}{c}\text { 6-12 } \\
\text { m }\end{array}$ & $\begin{array}{c}\text { 3-6 } \\
\text { m }\end{array}$ & $\begin{array}{c}6-12 \\
\mathrm{~m}\end{array}$ & \begin{tabular}{|c|}
$3-6$ \\
m
\end{tabular} & $\begin{array}{c}6-12 \\
m\end{array}$ & $\begin{array}{c}\text { 3-6 } \\
\text { m }\end{array}$ & $\begin{array}{c}6-12 \\
\text { m }\end{array}$ & & $\begin{array}{c}3-6 \\
\text { m }\end{array}$ & \begin{tabular}{|c|}
$6-12$ \\
m
\end{tabular} & \begin{tabular}{|c|}
$3-6$ \\
$\mathrm{~m}$
\end{tabular} & \begin{tabular}{|c|}
$6-12$ \\
$m$
\end{tabular} \\
\hline & 1.00 & 2.80 & 3.00 & 2.30 & 4.50 & 2.80 & 4.00 & 1.75 & 1.50 & 1.25 & 5.00 & 0.00 & 0.00 \\
\hline & 0.00 & & 1.50 & & 0.50 & & 1.25 & 0.75 & & 0.00 & 17.00 & .25 & \\
\hline & 0.50 & 0.00 & 0.00 & 0.00 & 3.50 & 0.00 & 0.00 & 0.25 & 0.00 & 0.00 & 0.00 & 0.00 & 0.00 \\
\hline & 0.00 & 0.00 & 3.00 & 0.50 & 0.50 & 0.00 & 0.50 & 1.25 & 2.00 & 5.25 & 5.25 & 0.00 & 0.00 \\
\hline & 6.50 & 10.75 & 17.00 & 7.30 & 5.50 & 3.00 & 4.50 & 11.25 & 8.50 & 7.50 & 10.25 & 1.00 & 2.50 \\
\hline & 0.50 & 3.50 & 3.50 & 0.00 & 0.00 & 1.50 & 0.50 & 5.50 & 2.00 & 6.75 & 9.75 & 0.00 & 1.25 \\
\hline & 28.80 & 1.30 & 57.50 & 7.50 & 13.80 & 2.30 & 10.25 & 4.50 & 58.25 & 39.00 & 10.25 & 0.00 & 0.00 \\
\hline Long- & 0.00 & 0.00 & 0.00 & 2.80 & 0.00 & 0.00 & 0.00 & 0.00 & 0.00 & 0.00 & 1.25 & 0.00 & 0.00 \\
\hline & 0.00 & 0.00 & 0.00 & 2.50 & 0.00 & 0.00 & 0.00 & 0.00 & 0.00 & 0.00 & 1.50 & 0.00 & 0.00 \\
\hline Black & 0.00 & 0.00 & 0.00 & 0.00 & 0.00 & 0.00 & 0.00 & 0.00 & 0.00 & 0.00 & 0.00 & 0.00 & 0.25 \\
\hline Short & 462.3 & 0.0 & 145.5 & 30.8 & 96.0 & 59.3 & 3.3 & 5.0 & 8.0 & 2.5 & 1.8 & 411.8 & 114.5 \\
\hline Long & & 123.5 & & 75.8 & 2.3 & 0.5 & 12.5 & & 3.0 & 3.3 & & 0.0 & 0.0 \\
\hline Penc & 0.0 & 0.0 & 13.3 & 32.0 & 0.0 & 0.0 & 0.5 & 2.3 & 0.0 & 0.0 & 0.0 & 0.0 & 0.0 \\
\hline & & & 0.0 & 0.0 & 2.0 & 1.8 & 0.8 & 2.3 & 0.0 & 1.0 & 1.5 & 0.0 & 0.0 \\
\hline & & & & 0.0 & 2.8 & 2.3 & 0.8 & 3.0 & 0.5 & 0.0 & 0.5 & 0.0 & 2.3 \\
\hline Triton shell & 0.0 & 0.8 & 7.0 & 7.3 & 0.0 & 0.0 & 0.0 & 0.0 & 0.5 & 0.8 & 0.0 & 0.0 & 0.0 \\
\hline
\end{tabular}

Table 6. Levels of anthropogenic impacts in 2007 transects

\begin{tabular}{|c|c|c|c|c|c|c|c|c|c|c|c|c|c|c|}
\hline \multirow[b]{2}{*}{$\begin{array}{l}\text { Anthropogenic } \\
\text { impact }\end{array}$} & \multirow{2}{*}{\begin{tabular}{|c} 
Hendorabi \\
$3-6$ \\
m
\end{tabular}} & \multirow{2}{*}{$\begin{array}{c}\text { Nayband1 } \\
3-6 \\
\text { m }\end{array}$} & \multirow{2}{*}{\begin{tabular}{|c|} 
Nayband2 \\
$3-6$ \\
$\mathrm{~m}$
\end{tabular}} & \multicolumn{2}{|c|}{ Kharku } & \multicolumn{2}{|c|}{ Khark } & \multicolumn{2}{|c|}{ Kish 1} & \multirow{2}{*}{\begin{tabular}{|c|} 
Kish2 \\
3-6 \\
m \\
\end{tabular}} & \multicolumn{2}{|c|}{ Farur } & \multicolumn{2}{|c|}{ Farurgan } \\
\hline & & & & $\begin{array}{l}3-6 \\
\text { m }\end{array}$ & \begin{tabular}{|c|}
$6-12$ \\
$m$
\end{tabular} & $\begin{array}{c}3-6 \\
m\end{array}$ & $\begin{array}{c}\text { 6-12 } \\
\text { m }\end{array}$ & $\begin{array}{l}3-6 \\
\text { m }\end{array}$ & $\begin{array}{c}\text { 6-12 } \\
\mathrm{m}\end{array}$ & & $\begin{array}{l}3-6 \\
\text { m }\end{array}$ & $\begin{array}{c}6-12 \\
\mathrm{~m}\end{array}$ & $\begin{array}{r}\text { 3-6 } \\
\text { m }\end{array}$ & $\begin{array}{c}6-12 \\
m\end{array}$ \\
\hline $\begin{array}{l}\text { Aquarium } \\
\text { fishing }\end{array}$ & 1 & 0 & 0 & 0 & 0 & 0 & 0 & 2 & 1 & 0 & 1 & 1 & 0 & 0 \\
\hline $\begin{array}{l}\text { Commercial } \\
\text { fishing }\end{array}$ & 1 & 3 & 3 & 1 & 1 & 2 & 2 & 0 & 2 & 1 & 1 & 1 & 0 & 0 \\
\hline Sewage & 0 & 0 & 0 & 0 & 0 & 0 & 0 & 0 & 0 & 3 & 0 & 0 & 0 & 0 \\
\hline $\begin{array}{l}\text { Industrial } \\
\text { pollution }\end{array}$ & 0 & 0 & 0 & 0 & 0 & 3 & 2 & 0 & 0 & 0 & 0 & 0 & 0 & 0 \\
\hline Tourist diving & 0 & 0 & 0 & 0 & 0 & 0 & 0 & 3 & 1 & 0 & 0 & 0 & 0 & 0 \\
\hline
\end{tabular}

Table 7. Average indicator fish/invertebrate density (individual/100 $\mathrm{m}^{2}$ ) in 2007 transects

\begin{tabular}{|c|c|c|c|c|c|c|c|c|c|c|c|c|c|c|}
\hline \multirow{2}{*}{$\begin{array}{c}\text { Fish/ } \\
\text { Invertebrate }\end{array}$} & \multirow{2}{*}{\begin{tabular}{|c|}
$\begin{array}{c}\text { Hendo } \\
\text { rabi }\end{array}$ \\
$3-6$ \\
m
\end{tabular}} & \multirow{2}{*}{\begin{tabular}{|c|} 
Nayband1 \\
$3-6$ \\
$m$ \\
\end{tabular}} & \multirow{2}{*}{\begin{tabular}{|c|} 
Nayband2 \\
$\begin{array}{c}3-6 \\
m\end{array}$ \\
\end{tabular}} & \multicolumn{2}{|c|}{ Kharku } & \multicolumn{2}{|c|}{ Khark } & \multicolumn{2}{|c|}{ Kish 1} & \multirow{2}{*}{\begin{tabular}{|c|} 
Kish2 \\
$\begin{array}{c}3-6 \\
\text { m }\end{array}$ \\
\end{tabular}} & \multicolumn{2}{|c|}{ Farur } & \multicolumn{2}{|c|}{ Farurgan } \\
\hline & & & & \begin{tabular}{|c|}
$3-6$ \\
m \\
\end{tabular} & \begin{tabular}{|c|}
$6-12$ \\
$\mathrm{~m}$ \\
\end{tabular} & $\begin{array}{c}3-6 \\
\mathrm{~m}\end{array}$ & $\begin{array}{c}6-12 \\
\mathrm{~m}\end{array}$ & \begin{tabular}{|c|}
$3-6$ \\
m
\end{tabular} & \begin{tabular}{|c|}
$6-12$ \\
$\mathrm{~m}$
\end{tabular} & & \begin{tabular}{|c|}
$3-6$ \\
m \\
\end{tabular} & $\begin{array}{c}6-12 \\
\mathrm{~m}\end{array}$ & $\begin{array}{c}3-6 \\
\text { m }\end{array}$ & $\begin{array}{c}6-12 \\
\mathrm{~m}\end{array}$ \\
\hline $\begin{array}{l}\text { Orange-spotted } \\
\text { grouper }\end{array}$ & 0.5 & 0.5 & 0.0 & 0.0 & 0.3 & 0.0 & 0.3 & 0.0 & 0.0 & 0.0 & 0.0 & 0.0 & 0.0 & 0.0 \\
\hline Other groupers & 1.5 & 0.8 & 0.8 & 0.3 & 0.3 & 0.0 & 0.0 & 0.5 & 0.0 & 0.0 & 0.0 & 0.5 & 0.3 & 0.3 \\
\hline Spotted grant & 0.0 & 0.0 & 0.0 & 0.0 & 0.0 & 0.0 & 0.0 & 0.0 & 0.0 & 0.0 & 0.0 & 0.0 & 0.3 & 0.3 \\
\hline Arabian butterfly & 0.5 & 0.0 & 0.0 & 0.0 & 0.3 & 0.0 & 0.0 & 5.5 & 0.0 & 0.0 & 2.5 & 0.0 & 0.5 & 0.5 \\
\hline Dark butterfly & 5.5 & 6.5 & 5.3 & 13.0 & 4.8 & 5.8 & 6.0 & 7.8 & 0.0 & 0.0 & 10.8 & 0.3 & 2.3 & 2.3 \\
\hline Parrot & 5.5 & 0.5 & 0.3 & 0.0 & 0.0 & 0.0 & 1.3 & 0.0 & 0.0 & 0.0 & 1.0 & 0.0 & 3.5 & 3.5 \\
\hline Moray & 0.0 & 0.0 & 0.0 & 0.0 & 0.0 & 0.0 & 0.0 & 0.0 & 0.0 & 0.0 & 0.0 & 0.3 & 0.0 & 0.0 \\
\hline Snapper & 0.8 & 25.8 & 1.8 & 0.0 & 0.5 & 0.0 & 0.0 & 0.0 & 0.0 & 0.0 & 0.0 & 0.0 & 0.0 & 0.0 \\
\hline $\begin{array}{l}\text { Short-spine sea } \\
\text { urchin }\end{array}$ & 1.3 & 0.0 & 700.8 & 270.8 & 155.5 & 60.8 & 0.0 & 0.0 & 0.0 & 65.5 & 0.0 & 22.8 & 1.0 & 0.0 \\
\hline $\begin{array}{l}\text { Long-pine sea } \\
\text { urchin }\end{array}$ & 0.0 & 0.0 & 0.0 & 13.3 & 62.5 & 0.0 & 58.8 & 0.0 & 2.3 & 13.0 & 58.8 & 18.5 & 0.0 & 0.0 \\
\hline Pencil sea urchin & 0.0 & 0.0 & 0.0 & 0.5 & 1.5 & 0.0 & 0.0 & 0.0 & 0.5 & 0.0 & 0.0 & 0.0 & 0.0 & 0.0 \\
\hline Cowry shell & 0.0 & 1.0 & 0.0 & 0.0 & 0.0 & 0.0 & 0.0 & 0.0 & 0.0 & 0.0 & 0.0 & 0.0 & 0.0 & 0.0 \\
\hline Sea cucumber & 0.5 & 0.0 & 0.0 & 0.3 & 0.0 & 0.0 & 0.0 & 0.0 & 0.3 & 0.0 & 0.0 & 0.0 & 0.3 & 0.0 \\
\hline
\end{tabular}




\section{Correlation between proposed indicators and anthropogenic variables}

Outliers of fish and invertebrate data in 2002, were moray eel and lobester, in 2003, were black-spotted grunt and cowry shells and in 2007 were moray eel and cowry shells.

DCA first axis gradients of fish and invertebrate data in 2002, were 2.174 and 0.782 , in 2003 were 1.237 and 1.893 and in 2007 were 1.695 and 1.944 respectively. Therefor redundancy analysis (RDA) was used for all cases.

In redundancy analysis for fish vs. anthropogenic impacts in 2002, dark butterfly fish, Arabian butterfly fish and to some extent hump-head wrass exhibited positive correlation and orange-spotted grouper and long-fin butterfly fish showed negative correlation with commercial fishing. Also hump-hed wrass showed positive correlation and parrot fish and other groupers showed negative correlation with aquarium fishing and tourist diving (Fig. 2). For invertebrates vs. anthropogenic impacts short-spine sea urchin, sea cucumbers and pencil sea urchin exhibited positive correlation with commercial fishing and to lower extent with aquarium fishing and negative correlation with tourist diving (Fig. 3).

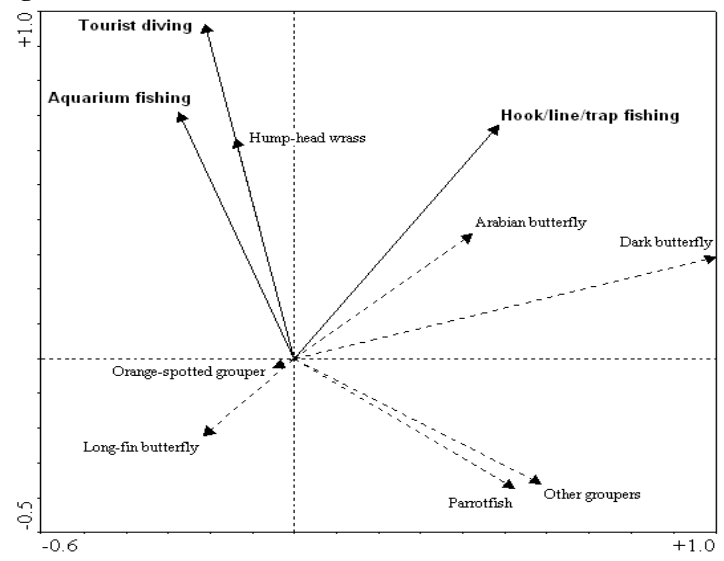

Figure 2. Redundancy analysis for fish vs. anthropogenic impacts in 2002

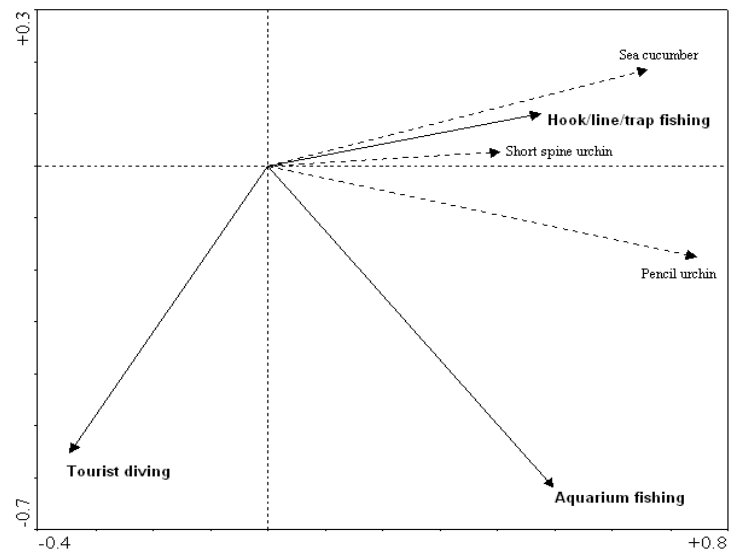

Figure 3. Redundancy analysis for invertebrate vs. .anthropogenic impacts in 2002

In redundancy analysis for fish vs. anthropogenic impacts in 2003, grey grut, long-fin butterfly fish, snappers, other groupers, Arabian butterfly fish, dark butterfly fish, orange-spotted grouper and parrot fish showed negative correlation with commercial 
fishing. In addition orange-spotted grouper, other groupers and spotted grunt showed positive correlation with tourist diving and Arabian butterfly fish, snappers, grey grut, dark butterfly fish, long-fin butterfly fish and parrot fish exhibited positive correlation with aquarium fishing and sewage pollution (Fig. 4).

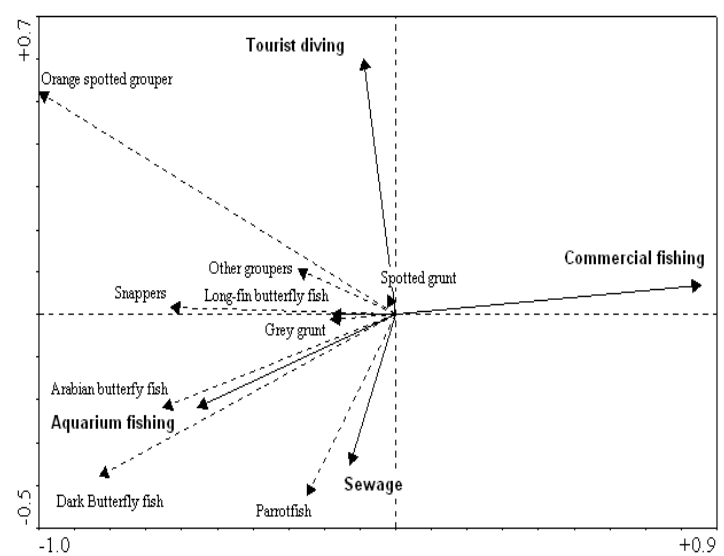

Figure 4. Redundancy analysis for fish vs.anthropogenic impacts in 2003

For invertebrates vs. anthropogenic impacts short-spine sea urchin exhibited positive correlation with commercial fishing and negative correlation with aquarium fishing, tourist diving and sewage pollution and sea cucumbers showed positve correlation with sewage pollution (Fig. 5). In addition short-spine sea urchin, triton shells, pencil sea urchin and long-spine sea urchin exhibited negative correlation with sewage pollution and long-spine sea urchin exhibited positive correlation with tourist diving.

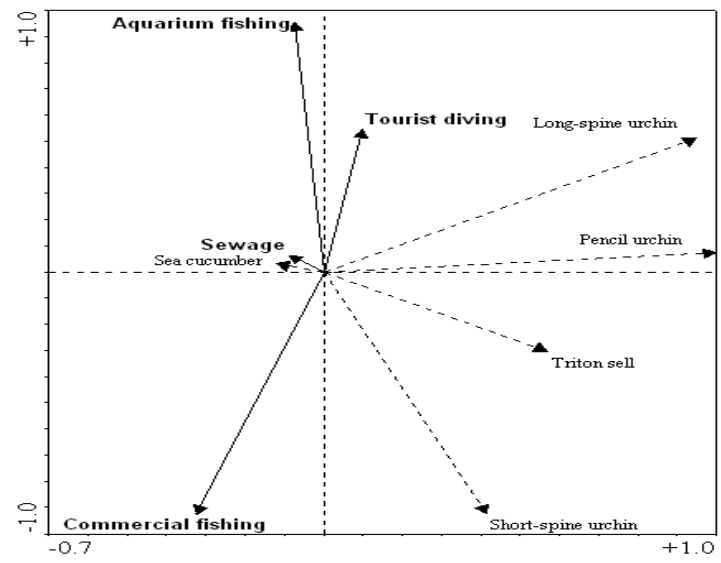

Figure 5. Redundancy analysis for invertebrate vs .anthropogenic impacts in 2003

In redundancy analysis for fish vs. anthropogenic impacts in 2007, Arabian butterfly fish, parrot fish and spotted grunt showed negative correlation and snappers, orangespotted grouper and other groupers showed more or less positive correlation with commercial fishing. Also Arabian butterfly fish, spotted grunt and parrot fish showed positive correlation, and snappers and orange-spotted grouper showed negative correlation with tourist diving, aquarium fishing and industrial pollution. In adition dark butterfly fish, orange-spotted grouper, other groupers, spotted grunt and parrot fish 
exhibited negative correlation with sewage pollution (Fig. 6). For invertebrates vs. anthropogenic impacts short-spine sea urchin and long-spine sea urchin exhibited positive correlation with commercial fishing and sewage pollution and negative correlation with tourist diving and aquarium fishing. Also pencil sea urchin showed negative correlation with industrial pollution and aqurium fishing and sea cucumbers showed positve correlation with tourist diving and negaitive correlation with commercial fishing and sewage pollution (Fig. 7).

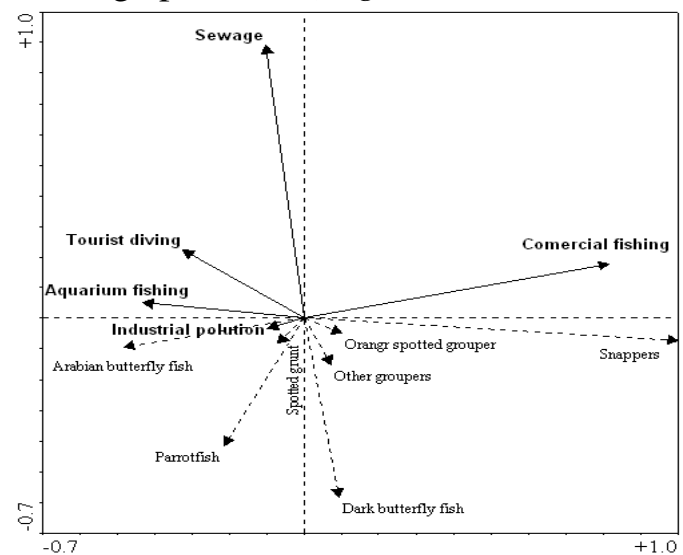

Figure 6. Redundancy analysis for fish vs .anthropogenic impacts in 2007

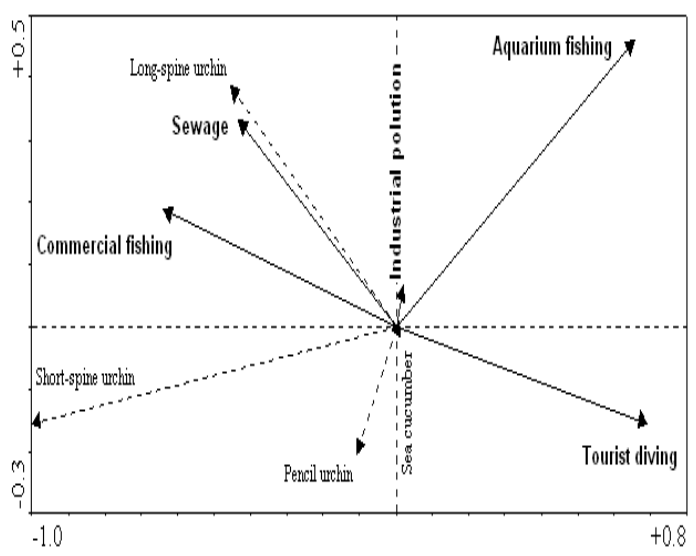

Figure 7. Redundancy analysis for invertebrate vs .anthropogenic impacts in 2007

\section{Indicator values of proposed indicators for different habitat groups}

Studied transects were clustered into groups based on anthropogenic impact types and levels. Caculated Indicator Values (IV)s for characteritic species/taxa of habitat groups recognized in the survey area in different years are presented in table 8 (Only indicator values $>60 \%$ and those with $\mathrm{p}<0.25$ are listed in this table). 
Table 8. Indicator Values for characteritic species/taxa of different anthropogenic impact groups/levels

\begin{tabular}{|c|c|c|c|c|}
\hline Species/Taxa & IV & $* \mathbf{P}$ & Habitat(Transect) roup & Year \\
\hline Dark butterfly & 97.0 & 0.2000 & & \multirow{2}{*}{2002} \\
\hline Arabian butterfly & 66.4 & 0.3980 & Commercial \& aquarıum tishıng & \\
\hline Orange-spotted grouper & 60.1 & 0.2370 & \multirow{5}{*}{ Mostly Aquarium fishing } & \multirow{9}{*}{2003} \\
\hline Other groupers & 69.2 & 0.1720 & & \\
\hline Arabian butterfly & 82.1 & 0.0190 & & \\
\hline Parrot fish & 80.5 & 0.0220 & & \\
\hline Cowries & 65.6 & 0.0830 & & \\
\hline Short-spine sea urchin & 87.0 & 0.0530 & \multirow[t]{2}{*}{ Commercial fishing } & \\
\hline Other groupers & 64.8 & 0.2720 & & \\
\hline Arabian butterfly & 71.81 & 0.0380 & \multirow[t]{2}{*}{ Low Commercial fishing } & \\
\hline Parrot fish & 67.5 & 0.0310 & & \\
\hline Spotted grunt & 66.7 & 0.0900 & & \multirow{4}{*}{2007} \\
\hline Arabian butterfly & 80.0 & 0.0420 & No Commercial fishing & \\
\hline Snappers & 98.5 & 0.0100 & High Commercial fishing & \\
\hline Short-spine sea urchin & 69.0 & 0.1010 & Commercial fishing & \\
\hline
\end{tabular}

\section{Discussion}

Looking at anthropogenic impacts, fishes and invertebrates proposed by Reef Check as Bio-indicators of coral reefs in the area are divided into five different groups:

1. Barramundi cod, hump-head wrasse, long-fin butterfly fish, banded coral shrimp, lobsters (all edible species), collector sea urchin, black sea urchin and pencil sea urchin.

2. Sea cucumbers, triton shells, cowries (all species) and lobesters (commomn with the first group).

3. Moray eel (all species) and banded coral shrimp (commomn with the first group).

Members of these three groups are not good indicators of coral reef ecosystems in the region because they are rare or not common, not widely distributed in the region, are nocturnal, hide in holes during the day and can not be counted easily, or they are proposed as indicators of harvest types (such as invertebrate collection for food/for curio) which are not used in the region, also they didn't show any consistent positive or negative correlation with anthropogenic variables such as harvest types, tourist diving, etc. Also they didn't show significant indicator values for different groups of harvesting types and other anthropogenic impact levels.

4. Orange-spotted grouper, other groupers, grey grunt, black-spotted grunt, spotted grunt, other grunts/Sweetlips, snappers, parrot fish, dark butterfly fish and long-spine sea urchin.

Members of this group exhibitted more or less negative correlation with commercial fishing but didn't show consistent significant indicator values for different groups of harvesting types and other anthropogenic impact and are not considered as reliable indicators for anthropogenic impacts in the region.

5. Short-spine sea urchin (Echinometra mathaei) and Arabian butterfly fish (Chaetodon melapterus).

Members of this group are commom, easy to count and widespread in the region and showed consistent correlation with commercial fishing and consistent high indicator 
values for commercial fishing habitats and could be considered as indicators of fishing status in coral reef ecosystems in the region.

Short-spine sea urchin exhibited positive correlations with commercial fishing in 2002, 2003 and 2007. Furthermore, Indicator Species Analysis showed them to be indicator species for commercial fishing areas, with indicator values of $87.0(p=0.053)$, $69.0(\mathrm{p}=0.10)$ respectively in 2003 and 2007, and therefore short-spine sea urchin could be considered as bio-indicator of fishing pressure in coral reef ecosystems of this region.

Wrasses (Labridae) and emperor (Lethrinidae) also present in the study region are reported as dominant sea urchin predators in kenyan marine protected areas by $[17,18$, 19]. Over-fishing and removing of these predator fishes could increase short-spine sea urchin population and high abundance of sea urchins results from reductions in sea urchin predator fishes.

Arabian butterfly fish showed heigh significant indicator values for none to low commercial fishing habitats in 2003 (IV = 82.1, p<0.05) and 2007 (IV = 80.0, p<0.05) and this is further illustrated by the RDA's where they have exhibited negative correlation with commerciale fishing in 2003 and 2007. However it appears that Arabian butterfly fish is a good indicator for coral reef habitats with none to low commercial fishing impact.

Although [7, 25] have suggested that corallivore butterfly fish could be used as indicator species for changing conditions of coral reefs and also relationship between temporal variation of butterfly fish population density and the corals was reported by [1, 27], however it dos not seem that commercial fishing and removal of finfish is having direct impact on Arabian butterfly fish population density, becaues it is not target fish in this types of harvest.

Studies indicate that removal of finfish is having the largest impact on reefs, and has a number of tertiary effects on other faunal groups and ecological processes [20].

It is councluded that increased sea urchins (e.g. Echinometra mathaei) poulation causes high bioerosion followed by decrease in live coral cover, and finally it is associated with a decrease in corallivore butterfly fish (e.g. Chaetodon melapterus) population.

Acknowledgements. We would like to express our appreciation to Mr Abolfazl Nikbakht and Mr Mahraz Shahnazi for helping us in fieldwork and Mr Omid Tabiei from Islamic Azad University of Arsanjan for preparing needed software. In addition, special thanks to Mr Amir Shomali Moghaddam and Mr Ghasem Nargesi members of the Kish Diving Centre, also Mr Mahmood Rezaei, Mr Mohammad Toulideh, Mr Abdolrahman moradzadeh and members of Bushehr provincial office in Nayband National park and Khark Island.

\section{REFERENCES}

[1] Adjeroud, M., Augustin, D., Galzin, R., Salvat, B. (2002): Natural disturbances and interannual variability of coral reef communities on the outer slope of Tiahura (Moorea, French Polynesia): 1991 to 1997. - Marine Ecology Progress Series 237: 121-131.

[2] Alcala, A.C., Gomez, E.D., Yap, H.T. (1987): Philippine coral reefs: status and human responses to changes. - Resource Management and Optimization 4: 297-340.

[3] Beyeler, S.C., Dale, V.H. (2001): Challenges in the development and use of ecological indicators. - Ecological Indicators 1: 3-10. 
[4] Brown, B.E. (1987): Worldwide death of corals: natural cyclic events or man - made pollution? - Marine Pollution Bulletin 18: 9-13.

[5] Coker, P., Kent, M. (1992): Vegetation description and analysis. - John Wiley and Sons Ltd, Chichester (UK).

[6] Coles, S.L., Fadlallah, Y.H. (1991): Reef coral survival and mortality at low temp. in the Arabian Gulf: new speciesspecific lower temperature limits. - Coral Reefs 9: 231-237.

[7] Crosby, M.P., Reese, E.S. (1996): A Manual for Monitoring Coral Reefs with Indicator Species: Butterfly fishes as Indicators of Changes on Indo-Pacific Reefs. - Office of Ocean and Coastal Resources Management, NOAA: Silver Spring, MD.

[8] Dahl, A.L., Salvat, B. (1988): Are human impacts, either through traditional or contemporary uses, stabilizing or destabilizing to reef community structure. - Proc. 6th Int. Coral Reef Symp.,Townsville 1: 63-69.

[9] Downing, N. (1985): Coral communities in an extreme environment: the northwest Arabian Gulf. - Proc 5th Int Coral Reef Congr, Tahiti 6 : 343-348.

[10] Dufrene, M., Legendre, P. (1997): Species assemblages and indicator species: The need for a flexible asymmetrical approach. - Ecological Monographs 67: 345-366.

[11] Fadlallah, Y.H., Allen, K.W., Estudillo R.A. (1995): Mortality of shallow reef corals in the western Arabian Gulf following aerial exposure in winter. - Coral Reefs 14: 99-107.

[12] Grigg, R.W., Dollar, S.J. (1990): Natural and anthropog. disturbances on coral reefs. - ln: Z. Dubinsky (cd.) Coral reefs: Ecosys. of the World 25, Elsevier, Amsterdam. 439-452.

[13] Hodgson, G., Kiene, W., Mihaly, J., Liebeler, J., Shuman, C., Maun, L. (2004): Reef Check instruction manual: a guide to Reef Check coral reef monitoring. - Reef Check, Institute of the Environment, University of California at Los Angeles.

[14] Kenchington, R.A., Hudson, B.E. (1988): Coral Reef Management Handbook. UNESCO, Jakarta, Indonesia.

[15] Kinsey, D.W. (1988): Coral reef system responses to some natural and anthropogenic stresses. - Galaxea 7: 113-128.

[16] Linton, D.M., Warner, G.F. (2003): Biological indicators in the Caribbean coastal zone and their role in integrated coastal manag. - Ocean and Coastal Manag. 46: 261-276.

[17] McClanahan, T.R. (1995): Fish predators and scavengers of the sea urchin Echinometra mathaei in Kenyan coral-reef marine parks. - Environm. Biology of Fishes 43: 187-195.

[18] McClanahan, T.R. (1999): Predation and the control of sea urchins and fleshy algae inthe patch reefs of Glovers Reef, Belize. - Ecosystems 2: 511-523.

[19] McClanahan, T.R., Muthiga, N.A. (1989): Patterns of predation on a sea urchin, Echinometra mathaei (de Blainville), on Kenyan coral reefs. - Journal of Exprimental marine Biology ecology 126(1): 77-94.

[20] McClanahan, T.R., Shafir, S.H. (1990): Causes and consequences of sea urchin abundance and diversity in Kenyan coral reef lagoons. - Oecologia 83: 362-370.

[21] McCune, B., Mefford, M.J. (1997): PC-ORD. Multivariate analysis of ecological data. MjM Software Design, Oregon.

[22] Palmer, M.W. (2004): Ordination methods- An overview. www.okstate.edu.

[23] Pauly, D., Chua, T.E. (1988): The overfishing of marine resources: Socioeconomic background in southeast Asia. - Ambio 17: 200-206.

[24] Pilcher, N.J., Wilson, S., Alhazeem, S.H., Shokri M.R. (2000): Status of coral reefs in the Arabian/Persian Gulf and Arabian Sea region (Middle East). - In: Status of Coral Reefs of the World: 2000, Wilkinson C (ed.) Australian Instit. of Marine Science, Townsville.

[25] Reese, E.S. (1981): Predation on corals by fishes of the family Chaetodontidae: implications for conservation and management of coral reef ecosystems. - Bulletin of Marine Science 31: 594-604.

[26] Salvat, B. (1987): Human Impacts on Coral Reefs: Facts and Recommendations. Antenne Museum E.P.H.E., Moorea, French Polynesia. 
[27] Shokri, M.R., Fatemi S.M.R., Crosby, M.P. (2005): The status of butterflyfishes (Chaetodontidae) in the northern Persian Gulf, I.R. Iran. - Aquatic Conservation: Marine and Freshwater Ecosystems 15: 91-99.

[28] Wilkinson, C. (2000): The 1997-98 mass coral bleaching and mortality event: 2 years on. - In: Status of Coral Reefs of the World: 2000, Wilkinson C (ed.). Australian Inst. of Marine Science, Townsville. 